\title{
Cyanotic in recovery: What seems safe maybe unsafe.
}

\author{
Ahmed Elnady, Dikshika Mathur. \\ The Royal Wolverhampton Hospitals NHS Trust, Wolverhampton, UK
}

\section{Background:}

Local anaesthetics (LA) can induce methaemoglobin (MetHb) formation with variable severity. Because hypoxia and cyanosis in recovery have a plethora of causes, diagnosis of LA-induced methaemoglobinemia could be quite challenging and hence delayed, especially when safe doses are adhered to.

\section{Case Report:}

A 78-kg ASA-II 45-year-old female, who had an upper limb day case surgery under regional block, was desaturating in recovery. She had a history of surgical removal of a brain tumor (20 years back), that left her with left hemiplegia, from which she recovered completely after 6 months of rehab.

An US-guided left axillary block (prilocaine 1\%, $35 \mathrm{ml}$ ) was given, augmented by mid-arm radial and forearm ulnar and median nerve blocks (L-bupivacaine $0.25 \%, 5 \mathrm{ml}$ each). Positioned right laterally, she had an uneventful surgery.

In $\mathrm{PACU}, \mathrm{SpO} 2$ was $82 \%$. High-flow oxygen therapy was started. Although initially pink, faint central and peripheral cyanosis started to develop. Examination revealed decreased air entry on left lung base. ECG was normal. CXR revealed raised left hemi-diaphragm and increased hilar bronchovascular markings (Fig.1).

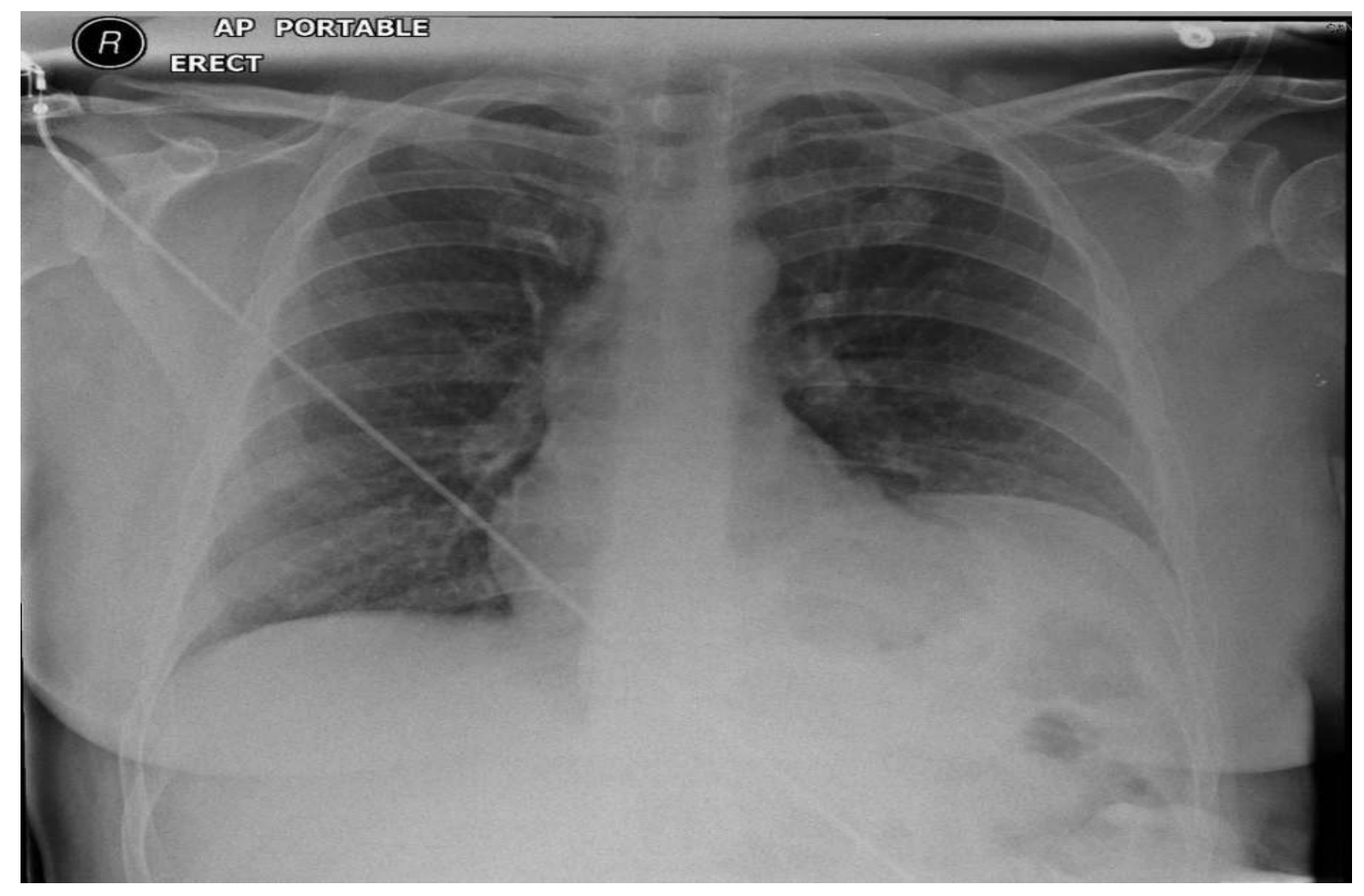

Figure 1: CXR of the patient showing raised left hemidiaphragm.

Apart from hypoxaemia, arterial blood gas $(A B G)$ results were normal. Co-oximetry showed metHb level of $13.1 \%$.

'Prilocaine-induced methaemogolbinemia' was the final diagnosis. She was kept under observation overnight for $\mathrm{O}_{2}$ therapy and serial ABGs. MetHb level dropped to $9.1 \%$ after 4 hrs and then $0.1 \%$ the next morning. At one month follow-up, CXR was normal.

\section{Discussion:}

Normally $1-2 \%$ of haemoglobin is metHb. Methaemoglobinemia, resulting from exposure to various chemicals including prilocaine's metabolite, O-toluidine, leads to oxidation of oxyHb into metHb, reducing $\mathrm{RBC}$ ability to release oxygen to tissues, and hence left shift of oxyHb dissociation curve.

Depending on metHb level, manifestations of progressive tissue hypoxia develop (see Table below). Of note, high levels of metHb tend to cause a pulse oximeter reading closer to $85 \%$ regardless of the true level of oxygen saturation.

\section{MetHb (\%)}

$1-2$

$<10$

$10-20$

20-30

$30-50$

$50-70$

$>70$

\section{Signs \& symptoms}

Normal

None

Peripheral and central cyanosis

Anxiety, tachycardia, headache

Confusion, dizziness, fatigue, tachypnea

Convulsions, coma, arrhythmias

Death
Diagnosis of methaemoglobinemia is based on clinical findings (i.e. oxygen-resistant cyanosis with normal $\mathrm{SpO}_{2}$ ) and confirmatory lab tests (co-oximetry is diagnostic).

\section{Treatment includes:}

1. Symptomatic: optimizing $\mathrm{O} 2$ delivery, respiratory and haemodynamic support.

2. Specific: methylene blue and/or ascorbic acid. (1)

Methylene blue (aka methylthioninum chloride) is given at 1-2 $\mathrm{mg} / \mathrm{kg}$ slowly IV over 5 minutes. A repeat dose of $1 \mathrm{mg} / \mathrm{kg}$ can be given after 1 hour.

Methylene blue's action is NADPH-dependent (Fig. 2). In glucose6-phosphate dehydrogenase [G6PD] deficiency, low levels of NADPH would make methylene blue not only ineffective, but also may cause haemolysis, hence it is contraindicated. In such case, ascorbic acid can be used, but clinical improvement would take longer.

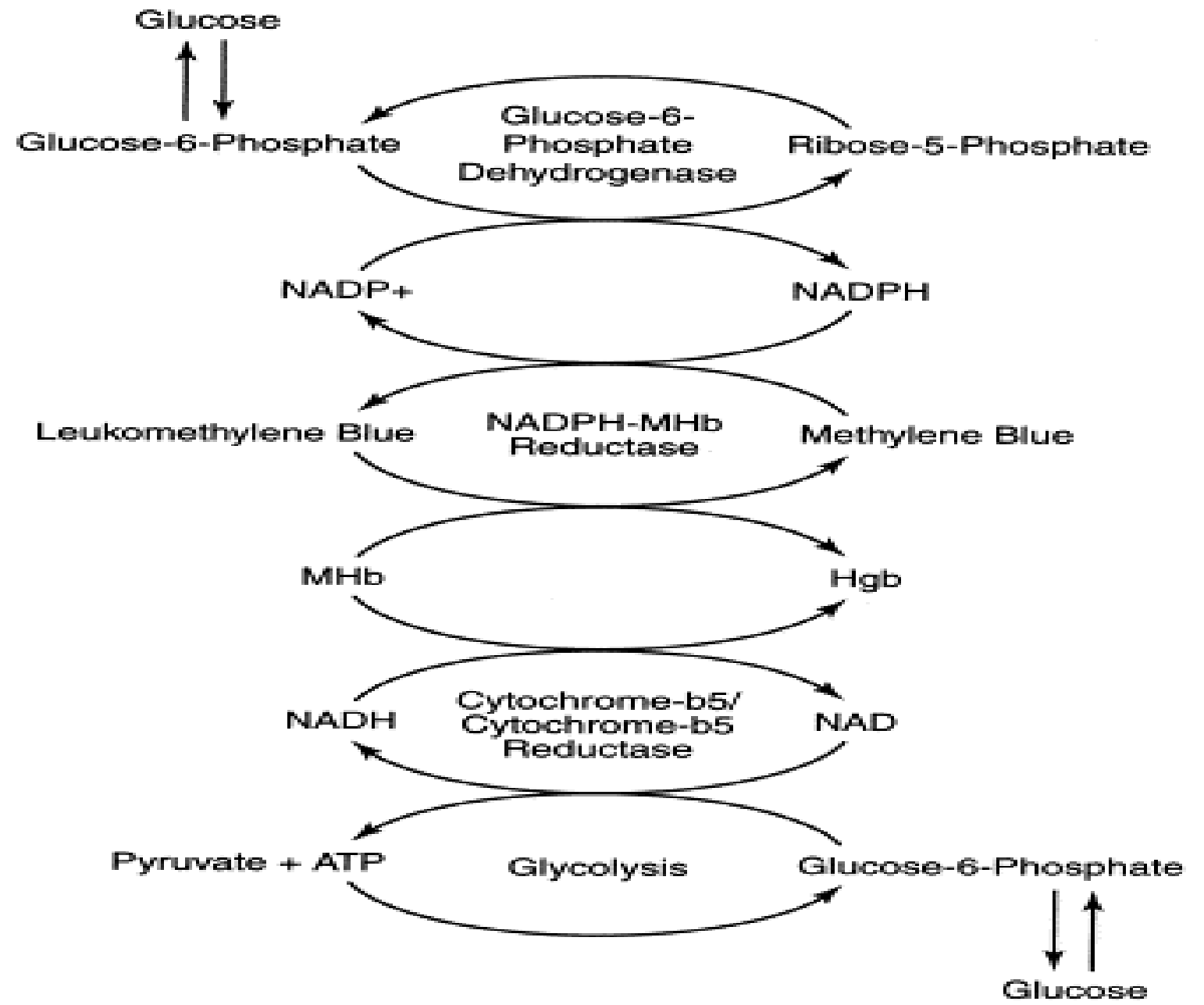

Figure 2: G6PD is pivotal for the NADPH-dependent methylene blue to convert metHb back to oxyHb.

In refractory methaemoglobinemia, hyperbaric oxygen or exchange transfusion may be used.

It is not generally appreciated that therapeutic doses of most LAs can produce this condition. (2) The maximum recommended dose of prilocaine is $6 \mathrm{mg} / \mathrm{kg}$. (3) Although this patient received a dose well below the maximum $(\sim 4.5 \mathrm{mg} / \mathrm{kg})$, she developed clinically significant methaemoglobinemia. She had no personal or family history of G6PD deficiency.

In 2009, Guay concluded that 'the dose of prilocaine should be limited to $2.5 \mathrm{mg} / \mathrm{kg}^{\prime}$. (1)

\section{Learning points:}

Prilocaine can induce methaemoglobinemia even in sub-maximal therapeutic doses. Caution should be exercised when using prilocaine in large volumes for major nerve blocks in small patients.

\section{References:}

1. Guay J. Methemoglobinemia related to local anesthetics: A Summary of 242 Episodes. Anesthesia \& Analgesia. 2009; 108(3):837-845.

2. Abu-Laban RB, et al. Severe methemoglobinemia from topical anesthetic spray... Can J Em Med. 2001; 3(1):51-56.

3. British National Formulary. September 2014: National Institute of Clinical Excellence (NICE). 